\title{
Dark Energy and Projective Symmetry
}

\author{
G. W. Gibbons ${ }^{1}$ and C.M. Warnick ${ }^{1,2}$ \\ 1. D.A.M.T.P., Cambridge, Wilberforce Road, Cambridge CB3 0WA, U.K. \\ 2. Queens' College, Cambridge, CB3 9ET, U.K.
}

November 1, 2018

\begin{abstract}
Nurowski arXiv:1003.1503 has recently suggested a link between the observation of Dark Energy in cosmology and the projective equivalence of certain Friedman-LemaitreRobertson-Walker (FLRW) metrics. Specifically, he points out that two FLRW metrics with the same unparameterized geodesics have their energy densities differing by a constant. From this he queries whether the existence of dark energy is meaningful. We point out that physical observables in cosmology are not projectively invariant and we relate the projective symmetry uncovered by Nurowski to some previous work on projective equivalence in cosmology.
\end{abstract}

\section{Introduction}

In order to set Nurowski's remark [1] in context we recall that the basic physical ideas motivating a projective viewpoint go back to the work of Neumann [2, Willam Thomson and Peter Tait 3], James Thomson [4, 5], Tait [6], Lange [7] and Muirhead [8] in the 19th century who re-examined the logical foundations of Newtonian Mechanics, and in particular the logical status of Newton's first law as recounted in in histories of mechanics [9, 10, 11, 12, 13. In order to give Newton's laws an operational meaning these authors introduced the idea of an inertial frame of reference and claimed that the free motion of four particles, not moving parallel to one another, defined a Cartesian coordinate system such that the motion of any other free particle would be given by a straight line in this coordinate system. That is the motion of freely falling particles endows space with a privileged set of unparameterized curves providing a projective structure and in the absence of forces this projective structure is flat.

The idea has been refined considerably since then. With the advent of special and general relativity, attention was transferred from space to spacetime 14, 15, 16. The world lines of freely falling particles define a projective structure on spacetime. This corresponds mathematically to a projective connection, i.e. an equivalence class of symmetric affine connections all having the same unparameterized auto-parallel curves

$$
\Gamma_{\mu}^{\nu}{ }_{\sigma} \equiv \Gamma_{\mu}^{\nu}{ }_{\sigma}+A_{\mu} \delta_{\sigma}^{\nu}+A_{\sigma} \delta_{\mu}^{\nu}
$$

Emails: g.w.gibbons@damtp.cam.ac.uk, c.m.warnick@damtp.cam.ac.uk

Pre-print no. DAMTP-2010-19 
However additional physical structures, such as idealised clocks or light rays are needed to justify the existence of a metric and relate it to the affine connection [17, 18, 19, and even more assumptions are required to obtain the dynamics of the metric.

Weyl introduced a projective curvature tensor 1 by

$$
W_{\mu \nu \sigma}^{\tau}:=R_{\mu \nu \sigma}^{\tau}+\frac{1}{n-1}\left(\delta_{\sigma}^{\tau} R_{\mu \nu}-\delta_{\nu}^{\tau} R_{\mu \sigma}\right) .
$$

and showed that a necessary and sufficient condition for two metrics to share the same geodesics, considered as unparameterized curves, is that their Weyl projective tensors, with this index structure, coincide. It follows that the vanishing of $W^{\tau}{ }_{\mu \nu \sigma}$ is a necessary and sufficient condition for the existence of a local coordinate chart, called a Beltrami chart, in which the geodesics are straight lines. A simple calculation using (2) then shows that the only such "projectively flat" spacetimes must have constant curvature, i.e. they must be Minkowski, de Sitter, or Anti-de Sitter spacetime. In Riemannian signature, this result is known as Beltrami's theorem. Thus these three spacetimes are on an equal footing from the purely projective point of view, just as they are on the same footing from the purely conformal point of view since all three are also conformally flat. Thus one can say that Newton's first law, in the form

Corpus omne perseverare in statu suo quiescendi vel movendi uniformiter in directum, nisi quatenus a viribus impressis cogitur statum illum mutare 2 .

holds in any one of these three spacetimes.

At a technical level, projective techniques were used by Eardley and Sachs 20] to provide spacetime with an ideal boundary: future projective infinity. More recently Twistorial and projective ideas have been applied to cosmology in 21. However in effect Hurd makes use of the conformal flatness of FLRW metrics rather than their truly projective properties. In that respect his work extends to the general FLRW case, the projective treatments of de Sitter spacetime of Du Val [14, 15].

At a late stage of this investigation we were made aware of the paper of Hall and Lonie 22 . which includes some of the results below, albeit without the focus on dark energy. We would like to thank M. Dunajski for drawing this work to our attention.

\section{Dark Energy and Projective Geometry}

We now turn to Nurowski's observation [1] and commence by subsuming it in a slightly more general result. This is 23 that a sufficient condition for two metrics to admit the same unparameterized geodesics, in the same coordinates $t, x^{i}$ is that if one is given by

$$
d s^{2}=-d t^{2}+a^{2}(t) g_{i j}(x) d x^{i} d x^{j},
$$

where $g_{i j}(x)$ is any metric depending only on the spatial coordinates $x^{i}$, then the other is

$$
d s^{2}=-\frac{d t^{2}}{\left(1-s a^{2}\right)^{2}}+\frac{a^{2}(t)}{1-s a^{2}} g_{i j}(x) d x^{i} d x^{j}
$$

The condition is also necessary for metrics of the form (3). Nurowski's result follows by taking if $g_{i j}$ to a metric of constant curvature $k$ in which both the metrics are of FLRW type. The more

\footnotetext{
${ }^{1}$ not to be confused with the Weyl conformal curvature tensor $C^{\tau} \mu \nu \sigma$

2 that is: All bodies tend to remain at rest, or to maintain a constant direction and speed, unless forced to do otherwise, by some external force.
} 
general result follows from the calculations in 23 by setting the $f$ of that reference to $\frac{1}{a^{2}}$. The fomulation of [23] in terms of $f$ makes it obvious the the projective transfomations form a one parameter symmery group acting as

$$
f \rightarrow f-s .
$$

It also follows from the calculations in 23, that while all FLRW metrics are conformally flat, i.e. their Weyl tensor vanishes, not all FLRW metrics are projectively flat. In fact as previously noted, the only FLRW metrics which are projectively flat are of constant curvature, that is they must, at least locally, be isometric with de Sitter, Minkowski or anti-de Sitter spacetime. Of course these are all (locally at least) projectively equivalent.

If we re-write the metric (4) as

$$
d s^{2}=-d \tau^{2}+A^{2}(\tau) g_{i j}(x) d x^{i} d x^{j},
$$

we have

$$
A^{2}=\frac{a^{2}}{1-s a^{2}}, \quad \Leftrightarrow \quad a^{2}=\frac{A^{2}}{1+s A^{2}} . \quad \Leftrightarrow \quad\left(1-s a^{2}\right)\left(1+s A^{2}\right)=1 .
$$

The time coordinates are related by

$$
d \tau=\frac{d t}{1-s a^{2}}, \quad \Leftrightarrow \quad d t=\frac{d \tau}{1+s A^{2}} .
$$

Note that if $s>0, a(t)$ runs from 0 to $+\infty$ in infinite $t$ time then $A(\tau)$ will run from from 0 to $+\infty$ in infinite $\tau$ time. On the other hand $A(t)$ will blow up in finite $t$ time and $a(\tau)$ will increase monotonically to $\frac{1}{s}$ in infinite $\tau$ time.

Differentiating the relation of (17) and making use of (8) we deduce that

$$
\frac{1}{a} \frac{d a}{d t}=\frac{1}{A} \frac{d A}{d \tau}
$$

Noting that (8) implies

$$
a^{2} \frac{d}{d t}=A^{2} \frac{d}{d \tau}
$$

we can further differentiate to find

$$
\begin{aligned}
a \frac{d^{2} a}{d t^{2}}-\left(\frac{d a}{d t}\right)^{2} & =A \frac{d^{2} A}{d \tau^{2}}-\left(\frac{d A}{d \tau}\right)^{2} \\
a^{3} \frac{d^{3} a}{d t^{3}}-a^{2} \frac{d^{2} a}{d t^{2}} \frac{d a}{d t} & =A^{3} \frac{d^{3} A}{d \tau^{3}}-A^{2} \frac{d^{2} A}{d \tau^{2}} \frac{d A}{d \tau} .
\end{aligned}
$$

Clearly these expressions are invariant under the projective transformation givexconstruct invariants of higher order in time derivatives. The second order invariant (11) appears in the components of the projective curvature of the metric (4). We find therefore that the Hubble expansion rate $H=\frac{1}{a} \frac{d a}{d t}$ is projectively invariant, however the deceleration $q=-a\left(\frac{d a}{d t}\right)^{-2} \frac{d^{2} a}{d t^{2}}$ and jerk $j=a^{2}\left(\frac{d a}{d t}\right)^{-3} \frac{d^{3} a}{d t^{3}}[24$ are not. The statements

$$
q=1, \quad \Leftrightarrow \quad a(t)=c_{1} e^{c_{2} t},
$$

and

$$
j=-q \quad \Leftrightarrow \quad a(t)=c_{1} \cos c_{2}\left(t-t_{0}\right), \quad \text { or } \quad a(t)=c_{1} \cosh c_{2}\left(t-t_{0}\right),
$$

\footnotetext{
${ }^{3}$ The reader is advised that relation between the coordinate $t$ and the coordinate $\tau$ depends on the parameter $s$. Thus the projective equivalence holds in the coordinates of (3) and (4), but not of (6).
} 
are projectively invariant and are obeyed by the projectively flat Minkowski and de Sitter metrics. In general these metrics may be characterised as having an energy density $\rho=c+d / a^{2}$ where $c$ and $d$ are constants.

If we assume the spatial metric to be of constant curvature then the Einstein equations imply the Friedman equations

$$
\begin{aligned}
\frac{\dot{a}^{2}}{a^{2}}+\frac{k}{a^{2}} & =\frac{8 \pi}{3} \rho \\
\frac{A^{\prime 2}}{A^{2}}+\frac{k}{A^{2}} & =\frac{8 \pi}{3} \tilde{\rho}
\end{aligned}
$$

where $\rho$ is the energy density of metric (3) and $\tilde{\rho}$ that of metric (4). A simple calculation, making use of (9), reveals the remarkable fact that

$$
\tilde{\rho}=\rho-\frac{3 k s}{8 \pi} .
$$

A brief calculation making use of the conservation equation shows that the pressure transforms according to

$$
\tilde{p}=\frac{p-s A^{2} \rho}{1+s A^{2}}+\frac{3 k s}{8 \pi} .
$$

The relation (17) suggests that a metric sourced by a pure Cosmological Constant $\Lambda$ is projectively equivalent under the transformation above to one with a Cosmological Constant $\tilde{\Lambda}$ given by

$$
\tilde{\Lambda}=\Lambda-3 k s .
$$

This can be seen explicitly. For example, we may take the $k=1$ metric of de Sitter

$$
d s^{2}=-d t^{2}+l^{2} \cosh ^{2}(t / l) d \Omega_{3}^{2}
$$

where $d \Omega_{3}^{2}$ is the metric on the unit 3 -sphere. This is the metric for the de Sitter space of constant curvature $\Lambda=3 / l^{2}$. From (8) we deduce that

$$
\frac{1}{l} \tanh \frac{t}{l}=\frac{1}{l^{\prime}} \tanh \frac{\tau}{l^{\prime}},
$$

where $\left(l^{\prime}\right)^{-2}=l^{-2}-s$. Making use of this, we find the identity

$$
A^{2}=\frac{l^{2} \cosh ^{2}(t / l)}{1-s l^{2} \cosh ^{2}(t / l)}=\left(l^{\prime}\right)^{2} \cosh ^{2}\left(\tau / l^{\prime}\right),
$$

so that the new metric is again de Sitter with a $k=1$ slicing, but with a new cosmological constant $\Lambda^{\prime}=3 /\left(l^{\prime}\right)^{2}=\Lambda-3 k s$. Similar calculations verify (19) directly in the case of de Sitter with $k=-1$ and anti-de Sitter with $k=-1$. For the $k=0$ slicings of de Sitter one finds that the transformation given above leads to exactly the same form of the metric. In this instance, the projective transformation is simply a diffeomorphism.

One might think that (17) implies that the projective transformation is equivalent to changing the matter content of the universe by adding a cosmological constant. It should be borne in mind, however, that the equation of state of the matter is not in general invariant under the projective transformations, because in in the FLRW metric (3) $\rho$ depends on $a$ while in the projectively equivalent metric (4) it depends on $A$. Thus for example if metric (3) is supported by matter with negligible pressure ("dust") then

$$
\rho=\frac{C}{a^{3}}, \quad \Rightarrow \quad \Lambda=0
$$


and so

$$
\begin{aligned}
& \tilde{\rho}=\frac{C}{A^{3}}\left(1+s A^{2}\right)^{\frac{3}{2}}-\frac{3 k s}{8 \pi}, \\
& \tilde{p}=\frac{3 k s}{8 \pi}-C \frac{s}{A}\left(1+s A^{2}\right)^{\frac{1}{2}}
\end{aligned}
$$

This differs from the result of Nurowski, who separates out a time-dependent cosmological constant in order that the matter content of the transformed metric take the form of dust with a cosmological constant. Since such a separation results in an energy-momentum tensor which is not conserved, we prefer to leave the energy-momentum intact.

For small $A$, i.e. for small $\tau$ time,

$$
\tilde{\Lambda} \approx 3 k s,
$$

but at late $\tau$ time

$$
\tilde{\Lambda} \approx-3 k s+8 \pi C s^{\frac{3}{2}} .
$$

in other words there is a effective $\tau$-time dependent Cosmological "Constant "as judged by the projectively equivalent metric (4).

\section{Physical Observables}

If

with

$$
d s^{2}=-d t^{2}+a^{2}(t)\left\{d r^{2}+f_{k}^{2}(r)\left(d \theta^{2}+\sin ^{2} \theta d \phi^{2}\right)\right\},
$$

$$
f_{k}(r)=\sin r, r, \sinh r, \quad \text { if } \quad k=1,0,-1,
$$

then a source emitting at $t=t_{e}$ is observed at $t=t_{o}$ with redshift

$$
1+z=\frac{a\left(t_{o}\right)}{a\left(t_{e}\right)} .
$$

Clearly the redshift is a physically measurable observable which is not invariant under the projective transformations (17).

The difference in the radial coordinate of source and observer is

$$
r\left(t_{o}\right)-r\left(t_{e}\right)=r=\int_{a\left(t_{e}\right)}^{a\left(t_{o}\right)} \frac{d a}{\sqrt{\frac{8 \pi}{3} \rho(a) a^{4}-k a^{4}}} .
$$

The intensity $I\left(t_{0}\right)$ received is

$$
I\left(t_{o}\right)=\frac{L\left(t_{e}\right)}{4 \pi} \frac{1}{f_{k}^{2}(r)(1+z)^{2}},
$$

where $L\left(t_{e}\right)$ is the total emitted luminosity. In astronomy it is traditional to define the magnitude $m\left(t_{o}\right)$ a source by the logarithm of the received intensity $I\left(t_{o}\right)$. Using equations (29)-(32) it one may construct a curve of $m$ against $z$ which is the redshift magnitude relation. This relation forms a major part of the evidence for the existence of dark energy. It is not invariant under projective transformations.

The angular size $\delta \phi$ of an sources of proper size $l_{e}$ is

$$
\delta \phi=\frac{l_{e}}{a\left(t_{e}\right) f_{k}(r)},
$$

and so similar remarks apply to the angular diameter redshift relation. 


\section{Conclusion}

As emphasised by Ehlers Pirani and Schild [17, spacetime requires more than just a projective structure if it is to describe the real world, since we can do more than just observe the (unparameterized) paths of freely falling particles. We can observe the motion of light rays, which determines a conformal structure and we can also measure, using atomic clocks, the lapse of proper time along the world lines of particles. This provides us with a preferred parameterization and in effect determines a pseudo-Riemannian structure. In the case of FLRW cosmology the metric structure is determined from, amongst other things, the observed redshift magnitude relation. As we have seen, this is not projectively invariant. The existence of dark energy is inferred, among other things, from the redshift magnitude relation, and this is also not projectively invariant.

Interestingly, the situation resembles a recent debate concerning whether the cosmological constant $\Lambda$ affects light bending, since the (unparameterized) projections of light rays in the static Schwarzschild-de Sitter or Kottler metric onto the spatial manifold do not depend on $\Lambda$ (see 23 and references therein). The origin of this phenomenon is that the projective structure of the associated optical metric is independent of $\Lambda$ 23. However this does not imply that there is no effect on observations of gravitational lensing since that involves measuring angles which are not invariant under projective transformations of the optical metric [23].

Nevertheless, while disagreeing with the suggestion [1] that "dark energy is meaningless" we believe that some intriguing connections have been uncovered which may conceivably lead to a deeper understanding of dark energy. It is striking that the reciprocal relations or dualities (77) recall those arising other contexts, such as Born-Infeld theory. Another interesting point relates to an old observation (see 24] and references therein) that much present day cosmological observations may be summarised by the statement that the universe is of FLRW type with jerk $j=1$. This implies that $k=0$ and that the universe was pressure free matter dominated at early times and dark energy dominated at late times. As we have seen, this is not a projectively invariant statement. But as we have also seen, the relations $q=-1$ and $j=-q$ are projectively invariant and are satisfied by solutions containing a cosmological constant and curvature energy.

\section{References}

[1] P. Nurowski, Is dark energy meaningless?, arXiv:1003.1503 [math.DG].

[2] C. Neumann, (1870). Ueber die Principien der Galilei-Newton'schen Theorie . Leipzig: B. G. Teubner, 1870 .

[3] W. Thomson and P. Tait Natural Philosphy 2nd edition; volume I (1879) pp 241-250

[4] J. Thomson, On the law of inertia; the principle of chronometry; and the principle of absolute clinural rest, and of absolute rotation. Proceedings of the Royal Society of Edinburgh 12 (1884) 568-78.

[5] J. Thomson, A problem of point motions for which a reference frame can so exists as to have motion of the points relative to it, rectilinear and mutually perpendicular Proceedings of the Royal Society of Edinburgh 12 (1884) 730-42.

(see Collected Papers of James Thomson ed J Larmor and J Thomson CUP (1912)

[6] P. G. Tait, Note on reference frames. Proceedings of the Royal Society of Edinburgh 12 (1884) 743-45. 
[7] L. Lange "Ueber das Beharrungsgesetz." Berichte uber Verhandlungen der Könglichen Sachsischen Gesellschaft der Wissenschaften zu Leipzig, Mathematisch-physische Classe $\mathbf{3 7}$ (1885): 333-51.

[8] R. F. Muirhead,The laws of motion. Philosophical Magazine, 5th series, 23(1887) : 473-89.

[9] E. Mach Die Mechanik in ihrer Entwickelung, historisch-kritisch dargestellt. 2nd edition. (1883) Leipzig: Brockhaus

[10] E. Mach, The Principles of Mechanics Open Court (1960)

[11] M. von Laue,History of Physics, Academic Press (1950)

[12] J. M. Jammer Concepts of Space, Dover

[13] J. Barbour The Discovery of Dynamics OUP (2001)

[14] P. Du Val, Geometrical Note on de Sitter's World Phil Mag 47 (1924) 930-938

[15] P. Du Val, On the discriminations between Past and Future Phil Mag 49 (1925) 379-390

[16] L. Silberstein, Projective Geometry of Galilean Space-Time Phil Mag 50 (1925) 681-696

[17] J. Ehlers, F. A. Pirani and A. Schild, The Geometry of Free Fall and Light propagation in General Relativity ed L. O' Rafeartaigh OUP (1972) 63-84

[18] J. Ehlers and A. Schild, Geometry in a manifold with a projective connection Comm Math Phys 32 (1973) 119-146

[19] U. Heilig and H. Pfister, Characterization of free fall paths by a global or local Desargues property J Geom Phys 7(1990) 419-446

[20] D. Eardley and R. K. Sachs, Spacetimes with a future projective infinity J Math Phys 14(1973)209-212

[21] T. R. Hurd, The projective geometry of simple cosmological models Proc Roy Soc London A 397 (1985) 233-243

[22] G. S. Hall and D. P. Lonie, The principle of equivalence and cosmological metrics, J. Math. Phys. 49 (2008) 022502

[23] G. W. Gibbons, C. M. Warnick and M. C. Werner, Light-bending in Schwarzschild-de Sitter: projective geometry of the optical metric," Class. Quant. Grav. 25 (2008) 245009 arXiv:0808.3074 [gr-qc]].

[24] M. Dunajski and G. Gibbons, Cosmic Jerk, Snap and Beyond,' Class. Quant. Grav. 25 (2008) 235012 arXiv:0807.0207 [gr-qc]]. 